\title{
EXPLANATION OF DIFFERENT SHAPES OF PLANETARIES \\ IN TERMS OF MOVEMENT IN MAGNETIC AND \\ GRAVITATIONAL FIELDS
}

\author{
E. Woyk (Elı̌̌Ka ChVojKová) \\ (Astronomical Institute, Czechoslovak Academy of Sciences, Czechoslovakia)
}

\begin{abstract}
Many features observed in solar prominences, planetary nebulae and the terrestrial magnetosphere (e.g. the filamentary structure, suspended clouds and arcs or toroids hovering above the equator) lead to the conviction that magnetic fields play a significant role in these objects. The magnetic mechanism (Woyk, 1967) described briefly below may be able to explain the shapes of all known planetaries. The field intensity sufficient for supporting the mechanism described need not exceed about $10^{-6}-10^{-4} G$.
\end{abstract}

\section{Theory of Particle Paths in Magnetic and Gravitational Fields}

\section{A. SPIRALLING IN A COLlisionless Plasma}

Besides the well-known magnetic-mirror level $r_{H}$, an upper reflecting level $r_{g}$ can also often arise due to gravitation (see Figure 1). The level $r_{g}$ is the highest altitude that a particle with a given initial path-radius and gyrofrequency can attain in a gravitational field. Thus in a collisionless plasma some particles should continually spiral up and down along the same field line, perpetually reflected between $r_{H}$ and $r_{g}$. At $r_{H}$ and $r_{g}$ the path is perpendicular to the magnetic field $H$; its deviation from $H$ is there $i=90^{\circ}$. The angle $i$ is a minimum at the level $r_{W}$ (characteristic for each path) at which the particle attains the critical velocity $v_{W}$. For a perfect dipole $v_{W}=v_{\text {esc }} / 3^{1 / 2}$ $\left(v_{W}\right.$ and the escape velocity vary with the distance $r$ from the centre). A particle reflected just when its velocity coincides with $v_{W}$ merely circles around the field line without any drift along or across it (dashed circles in Figure 1). Otherwise, below $r_{W}$ the particle velocity $v$ is higher than $v_{W}$ (higher even than the local $v_{W}$ corresponding to the investigated point), above $r_{W}$ the particle is slower.

\section{B. EFFECT OF ELASTIC COLLISIONS}

Let us deal with a plasma cloud emerging from below the magnetic surface (i.e. from below the level where the magnetic pressure $p_{H}$ exceeds the kinetic pressure, 


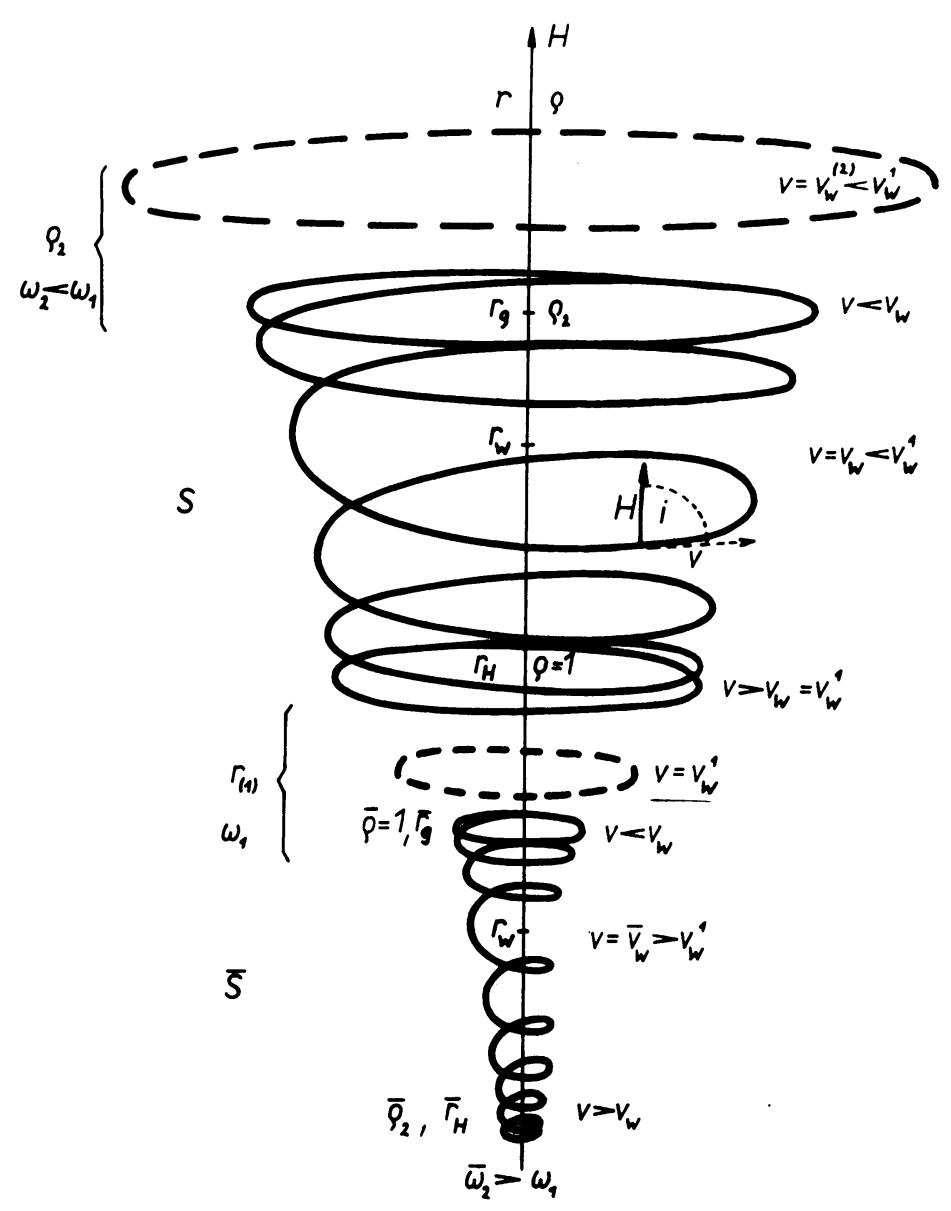

FIG. 1. Spiralling of charged particles along magnetic-field lines in an extended gravitational field, neglecting collisions. Particles reflected with the critical velocity $v_{W}$ can merely circle around $H$ (dashed lines); faster particles spiral below $r_{W}$, slower above $r_{W}$. The necessary path formulae are summarized by Woyk (1967) or Chvojková (1965).

$p_{\mathrm{kin}}$, and where the gyrofrequency $\omega_{H}$ is higher than the collision frequency). With regard to the velocity distribution the plasma cloud should spread out along the field line and disappear below the magnetic surface again (either at the same point or at the magnetically conjugate point). At high altitudes, however, where particles slide rather than spiral along the field line (where $i \approx 0$ ), elastic collisions contribute to an increase of $i$, and therefore the mean $\bar{r}_{H}$ and $\bar{r}_{g}$ shift closer to each other. When the lower reflecting level $\bar{r}_{H}$ has emerged above the surface the remaining plasma can no longer escape below the surface. Apparently immobile clouds, arcs or bunches are thus created in spite of an extremely high inner particle velocity, com- 
parable with the escape velocity. Clouds in which the mean particle velocity $\bar{v}>v_{W}$ tend to accumulate closer to the top of the magnetic loops, while clouds with lower mean particle velocity $\bar{v}$ tend to accumulate nearer their bottom.

\section{Application to Planetary Nebulae}

The shapes of Figure 2 assume that a uniform burst of plasma occurs from the whole surface. The expanding plasma tears magnetic-field lines from the central star, so that the resulting field $\mathrm{H}=\mathrm{Cr}^{-n}$ with $n \ll 3$ is far different from a magnetic-dipole

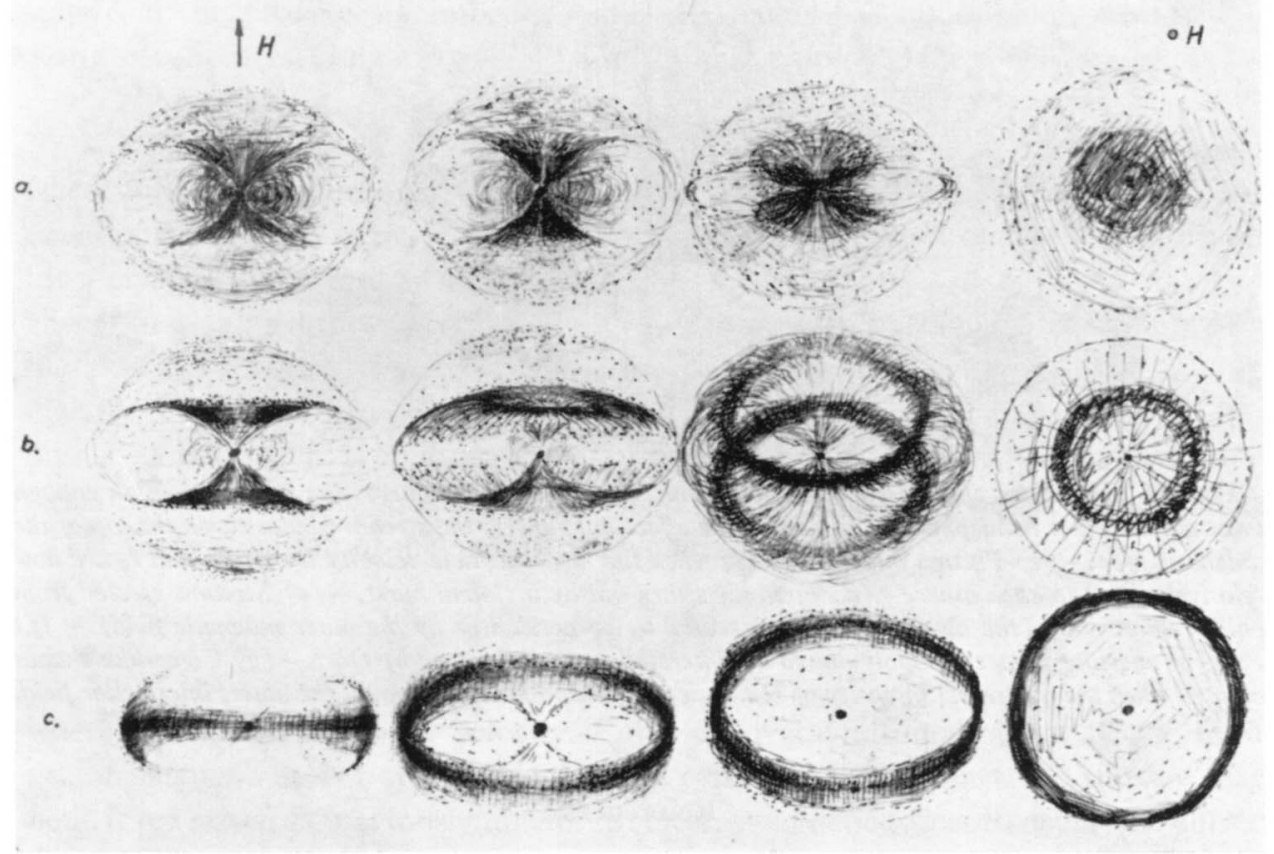

FIG. 2. Shapes arising from uniform plasma bursts ejected from the whole stellar surface. (a) The mean particle velocity is slow (then the mean $\bar{r}_{W}$ is close to the bottom of the field lines, and also the plasma of the polar caps has been trapped between $\bar{r}_{H}$ and $\bar{r}_{g}$ ). - (c) High (over escape) velocity of spiralling particles (accumulation at the top of magnetic loops; polar caps have escaped into the interstellar space). Left: equatorial view; right: polar view.

field. Plasma streams bursting from equatorial regions disappear at the conjugate points, producing a relatively hollow cave within the more dense outer-plasma toroid in which - according to collisions and $\bar{v}$ - a considerable remainder of the initial plasma jet becomes trapped, either at the top or at the bottom of the magnetic-field lines. (The diffusion along or across $H$ produced by elastic collisions is very slow). 
Figure 3 deals with some non-uniform effects. When the increase of density has led to $p_{\text {kin }}>p_{H}$, the magnetic-field system collapses. Also a very violent burst can produce the most irregular shapes with distorted broken filaments (Crab Nebula).

The upper model is not regarded as final. The aim of this contribution is only to demonstrate that the magnetic field should not be regarded as insignificant as has been done up to now.

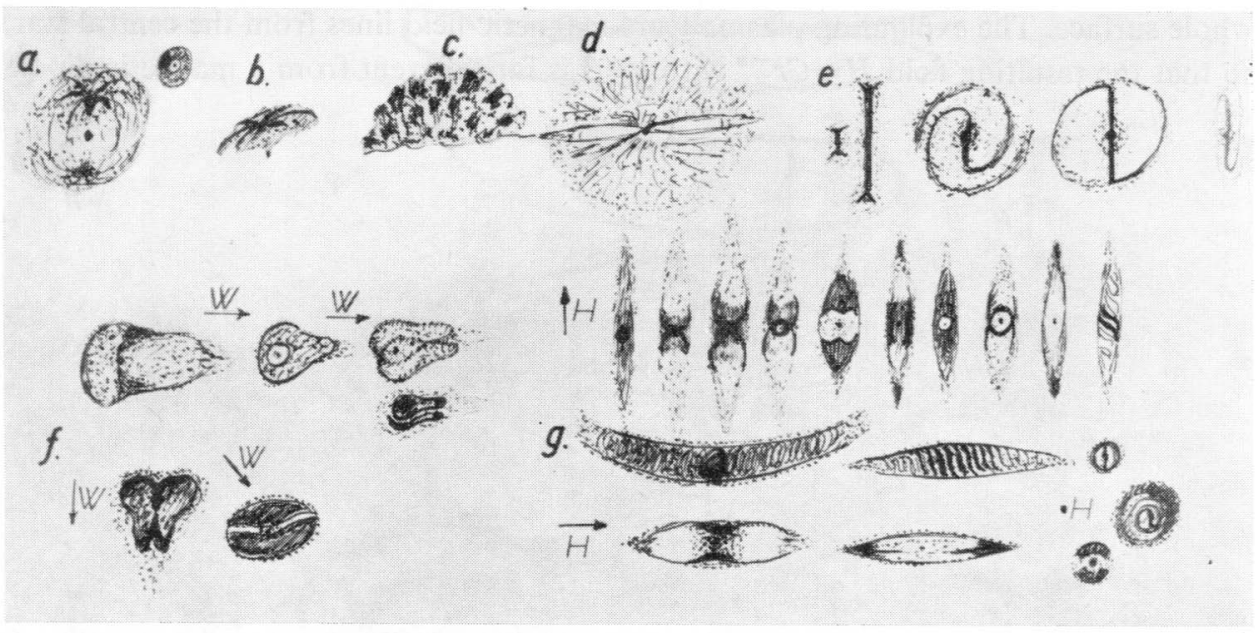

FIG. 3. Secondary effects: (a) Ejection from preferred active centres; the velocity is high enough to attain the other hemisphere. - (b) The same effect but slower; the stream cannot reach the top of the magnetic loop. - (c) Plasma bunches arising when the mean particle velocity is $v_{W} ; \bar{r}_{H}$ and $\bar{r}_{g}$ are now relatively close to each other. - (d) Field lines torn out by a violent burst. - (e) Streams ejected from polar regions only (the distortion can be created by precession or by the outer magnetic field). $-(f)$ Pear-or egg-like shapes probably due to the interstellar wind observed by Oort. - (g) Cigar-like shapes created when the outermost intensity of $H$ has decreased so much as to equal the outer, interstellar field.

\section{References}

Chvojková, E. (1965) Bull. Astr. Inst. Čsl., 16, 63.

Chvojková, E., Klepešta, J. (1965) Bull. Astr. Inst. Čsl., 16, 70.

Woyk, E. (1967) J. atmos. terr. Phys., 29, 465.

\section{DISCUSSION}

Flower: Would you expect to see synchrotron radiation from these objects?

Woyk: Yes, I think so.

Aller: Could a field of the expected magnitude be detected from the Zeeman effect of the $109 \alpha$ line?

Terzian: I do not think that we can observe the Zeeman effect in the $\mathrm{H} 109 \alpha$ line if the magnetic fields are as low as $10^{-5}$ gauss. 